\title{
Operative Vaginal Delivery Is a Safe Option in Women Undergoing a Trial of Labor after Cesarean
}

\author{
Erin Krizman, MD ${ }^{1}$ Patricia Grzebielski, MD ${ }^{1}$ Kathleen M. Antony, MD, MSCI ${ }^{1 \odot}$ \\ Emmanuel Sampene, $\mathrm{PhD}^{1}$ Matthew Shanahan, MD ${ }^{2}$ J. Igor Iruretagoyena, MD, MS ${ }^{1}$ Justin Bohrer, MD \\ ${ }^{1}$ Division of Maternal-Fetal Medicine, Department of Obstetrics and \\ Gynecology, University of Wisconsin School of Medicine and Public \\ Health, Madison, Wisconsin \\ 2 Department of Obstetrics and Gynecology, Barnes-Jewish Hospital, \\ Washington University in St. Louis, St. Louis, Missouri

\begin{abstract}
Address for correspondence Kathleen M. Antony, MD, Division of University of Wisconsin School of Medicine and Public Health, 4th Floor McConnell Hall, 1010 Mound Street, Madison, WI 53715
\end{abstract} \\ Maternal-Fetal Medicine, Department of Obstetrics and Gynecology, \\ (e-mail: kantony@wisc.edu).
}

Am J Perinatol Rep 2019;9:e190-e194.

\begin{abstract}
Keywords

- trial of labor after cesarean

- operative vaginal delivery

- forceps delivery

- vacuum-assisted delivery

- vaginal birth

- cesarean delivery

Objective To compare outcomes of operative intervention in the second stage of labor during trial of labor after cesarean (TOLAC).

Study Design A secondary analysis of the Maternal-Fetal Medicine Units Network cesarean section registry was conducted. Analysis was by first attempted mode of delivery. Results A total of 1,837 met inclusion criteria. Subjects in the operative vaginal groups (OVDs) were more likely to have a prior vaginal delivery (vacuum 34.2\%; forceps $34.3 \%$ ) than the repeat cesarean delivery (RCD) group (22.6\%; $p<0.0001)$. Most OVD attempts were successful (forceps $90.4 \%$; vacuum $92.6 \%$ ). Neonatal morbidity was not different ( $12.1 \%$ forceps vs. $14.6 \%$ vacuum; $14.8 \%$ RCD). Maternal morbidity was highest among forceps deliveries ( 32.3 vs. $24.3 \%$ vacuum; $22.0 \% R C D, p=0.0001$ ). RCD was associated with surgical injury ( 2.7 vs. $0.7 \%$ forceps; $0 \%$ vacuum; $p<0.0001$ ), endometritis ( 8.4 vs. $3.2 \%$ forceps, $1.2 \%$ vacuum; $p<0.0001$ ), and wound complications ( 1.9 vs. $0.4 \%$ forceps; $0.3 \%$ vacuum; $p=0.006$ ). OVD was associated with anal sphincter laceration ( $22.7 \%$ forceps, $15.5 \%$ vacuum; $0 \% R C D ; p=0.01$ ).

Conclusion The success rate of OVD is high in TOLAC with similar outcomes to RCD. Maternal composite outcomes were highest with forceps-assisted vaginal deliveries. However, considering overall morbidity, OVD in the second stage of labor in TOLAC is a reasonable, safe option in selected cases.
\end{abstract}

There has been a drastic increase in cesarean delivery rates in the United States over the past 40 years, such that $32 \%$ of all deliveries were via cesarean in 2015 , compared with $5 \%$ in $1970 .^{1-3}$ Cesarean deliveries are associated with increased morbidity and mortality as well as risks for future pregnancies including uterine rupture, abnormal placentation, and increased risks during subsequent abdominal surgeries. The risk of placenta accreta or previa, surgical injury (cystotomy, bowel injury, ureteral injury), ileus, intensive care unit (ICU)

\footnotetext{
D Kathleen M. Antony's ORCID is https://orcid.org/0000-0003$3537-8030$
}

received

July 2, 2018

accepted after revision

April 12, 2019
DOI https://doi.org/

$10.1055 / \mathrm{s}-0039-1692482$. ISSN 2157-6998. admission, hysterectomy, and blood transfusion requiring four or more units has been shown to be significantly increased with increasing number of cesarean deliveries. ${ }^{4}$ To avoid these increased risks, women who have had a prior C-section may opt for a trial of labor after cesarean (TOLAC) versus a repeat $\mathrm{C}$-section.

Women who elect for TOLAC may need operative intervention during the second stage of labor. Indications for operative intervention occur in $\sim 14 \%$ of women in the second stage of labor. The most common indication for intervention is nonreassuring fetal status followed by prolonged second stage. ${ }^{5}$ Options to terminate the second stage of labor include repeat

Copyright @ 2019 by Thieme Medical Publishers, Inc., 333 Seventh Avenue, New York, NY 10001, USA. Tel: +1(212) 584-4662.
License terms

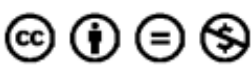


cesarean delivery (RCD) or instrumental delivery with vacuum or forceps (operative vaginal delivery). Operative vaginal delivery is one way in which a cesarean delivery can be avoided. However, operative vaginal deliveries have declined since 1990 from 9.01 to $3.30 \%$ in $2013 .^{6}$ This decline is likely multifactorial but mostly due to decreased training in residency. There have been a few studies looking at delivery options during the second stage of labor but little data specifically on the TOLAC population.

A recent study examined term pregnancies with no prior vaginal delivery and compared outcomes in vacuum-assisted deliveries, forceps-assisted deliveries, and cesarean deliveries. ${ }^{7}$ They found that attempt at vacuum delivery was associated with the lowest frequency of maternal complications except for severe lacerations which was lowest in the cesarean delivery group. Forceps and vacuum deliveries were associated with fewer postpartum infections. ${ }^{7}$

The goal of this study is to determine the best route of delivery in the second stage of TOLAC by comparing maternal and neonatal outcomes associated with cesarean, vaginal forceps, and vaginal vacuum deliveries based on the first attempted mode of delivery.

\section{Study Design}

Institutional Review Board exemption for secondary analysis of existing data was obtained. Data were collected from the Maternal-Fetal Medicine Units (MFMU) Network cesarean section registry. The MFMU registry includes women who had a pregnancy at 20 weeks or more of gestation or whose infant weighed at least $500 \mathrm{~g}$. Data were prospectively collected between 1996 and 2002 from 19 academic medical centers. Data were extracted directly from medical records by trained study nurses. Neonatal data were collected up to 120 days following delivery or at the time of hospital discharge. ${ }^{8}$

A cohort from this database was made which included women with gestational age more than 37 weeks, singleton pregnancy, estimated fetal weight more than $2,500 \mathrm{~g}$, and history of one prior cesarean delivery. Patients were included only if they achieved complete cervical dilation. Patients undergoing planned elective RCD were excluded as well as patients with multiple prior cesarean $(>1)$. Stillbirths, major fetal anomalies, noncephalic presentation, mid- or high forceps, patients with sequential vacuum-forceps or high forceps were also excluded. ${ }^{7}$ Fetal station at the time of intervention was not available through this database (see - Fig. 1).

Maternal and neonatal outcomes were compared in this cohort of women with one prior cesarean between first attempted mode of delivery in the second stage (vacuum, forceps, or repeat cesarean). Differences in the frequency distributions of baseline covariates of women with prior cesarean delivery undergoing operative intervention in the second stage of labor were compared via chi-square test or Fisher's exact test for categorical variables and $t$ test for continuous variables. There were two composite end points: maternal composite morbidity and neonatal compo- site morbidity. The maternal composite morbidity includes uterine rupture/dehiscence, surgical injury (enterotomy, cystotomy, other), death, endometritis, transfusion, hysterectomy, thromboembolism, wound complication, and anal sphincter lacerations. Also, neonatal composite morbidity included neonatal ICU (NICU) admission, hypoxic-ischemic encephalopathy (HIE), sepsis, Apgar $<4$ at 5 minutes, perinatal death, and birth injury. These composite end points were compared among the three groups of vacuum, forceps, and repeat $\mathrm{C}$-section methods of delivery using chi-square statistics. We report the success rates for each of these groups and analyses are interpreted in terms of odds ratios. All analyses were done at the $\alpha$ significance level of 0.05. Data analysis was performed using Stata Statistical Software: Release 14 (StataCorp LP; College Station, TX).

\section{Results}

There were a total of 73,257 deliveries over the study period in the cesarean section registry. Of these, 1,837 had an operative delivery after reaching the second stage of labor and thus were included in our study. There were 608 patients undergoing vacuum attempts, 743 forceps attempts, and 486 RCDs in the second stage of labor (-Fig. 1).

Baseline characteristics between operative vaginal deliveries (vacuum and forceps) and RCD were similar, with no significant difference between the groups in terms of obesity, diabetes, chronic hypertension, and pre-eclampsia. The average age of the patient was 29 years. The overall mean gestational age at delivery was $\sim 39^{5 / 7}$ weeks (39.7) (-Table 1). However, subjects undergoing forceps deliveries were more likely to be non-Hispanic black or Hispanic. Additionally, subjects in the operative vaginal delivery groups were more likely to have had one or more prior vaginal delivery (vacuum $34.2 \%, n=208$; forceps $34.3 \%$, $n=255)$ than the RCD group (22.6\%, $n=110 ; p<0.0001)$.

Most operative vaginal deliveries were successful (forceps $90.4 \%, n=672$; vacuum $92.6 \%, n=563$ ).

Maternal composite morbidity was highest among forceps deliveries ( $32.3 \%, n=240$ vs. $24.3 \%, n=148$ vacuum; $22.0 \%, n=107 \mathrm{RCD}, p=0.0001)$. Cesarean delivery was associated with a higher incidence of surgical injury $(2.7 \%$, $n=13$ vs. $0.7 \%, \quad n=5$ forceps; $0 \%, n=0$ vacuum; $p<0.0001$ ), endometritis (8.4\%, $n=41$ vs. $3.2 \%, n=24$ forceps, $1.2 \%, n=7$ vacuum; $p<0.0001$ ), and wound complications $(1.9 \%, n=9$ vs. $0.4 \%, n=3$ forceps; $0.3 \%, n=2$ vacuum; $p=0.006)$. Forceps delivery was associated with an increased risk of blood transfusion (3.4\%, $n=25$ vs. $1.3 \%$, $n=8$ vacuum; $1.9 \%, n=9 \mathrm{RCD} ; p=0.03)$. Operative vaginal delivery was associated with an increased risk of anal sphincter laceration (22.7\%, $n=169$ forceps, $15.5 \%, n=94$ vacuum; $0 \%, n=0$ RCD; $p=0.01$ ) (-Table 2 ).

Neonatal composite morbidity was not different among groups $(12.1 \%, n=90$ forceps vs. $14.6 \%, n=89$ vacuum; $14.8 \%, n=72 \mathrm{RCD})$. There was no difference in NICU admissions $(11.2 \%, n=68$ vacuum vs. $8.1 \%, n=60$ forceps; $11.9 \%$ $n=58 \mathrm{RCD}, p=0.0 .322)$, $\operatorname{HIE}(0.2 \%, n=1$ vacuum vs. $0.3 \%$, $n=2$ forceps; $0.4 \% n=2$ RCD, $p=0.0 .738)$, Apgar $<4$ at 


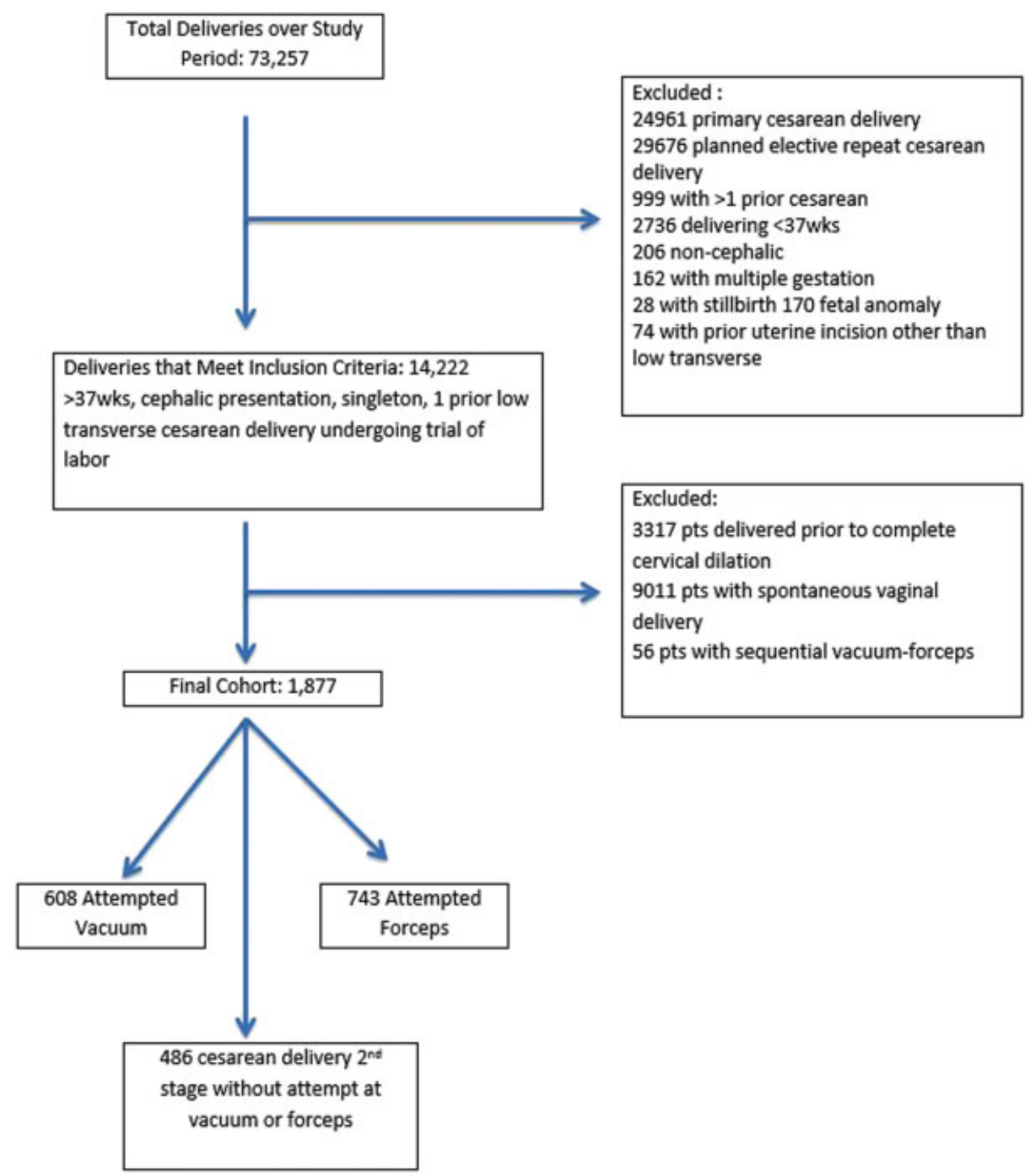

Fig. 1 Flow diagram for patient selection.

5 minutes $(0.5 \%, n=3$ vacuum vs. $0.8 \%, n=6$ forceps; $0.4 \%$, $n=2 \mathrm{RCD}, p=0.738)$, perinatal death $(0 \%, n=0$ vacuum vs. $0.1 \%, n=1$ forceps; $0 \%, n=0$ RCD, $p=0.0 .478)$, birth injury ( $2.8 \%, n=17$ vacuum vs. $2.8 \%, n=21$ forceps; $2.3 \%, n=11$ RCD, $p=0.0 .812$ ) (-Table 3 ).

\section{Conclusion}

In our analysis, the success rate of operative vaginal delivery in the setting of TOLAC is high with similar neonatal and maternal outcomes to second-stage RCD suggesting this is a safe option. Operative vaginal delivery was associated with a lower incidence of surgical injury, wound complications, uterine rupture, and endometritis but with a higher incidence of anal sphincter laceration. Maternal composite morbidity was highest among forceps deliveries, with an increased risk of blood transfusion. There was no difference in the composite neonatal outcomes.

Our findings are consistent with a recent study by Son et $\mathrm{al}^{9}$ demonstrating no increased maternal or neonatal adverse outcomes with operative vaginal delivery compared with second-stage RCD but with decreased endometritis risk. Our study was able to include a larger sample in the analysis (600 more women) as we included all women achieving complete dilation who did not have a mid-forceps delivery (the prior study was limited to women achieving a documented +2 station $)$.

This analysis is strong because of its large cohort size, multicenter nature, and prespecified, clinically relevant outcomes. Additionally, analysis was by first attempted mode of delivery, essentially an intention to treat, which decreases potential bias.

There are multiple limitations to this study including the retrospective nature, though a randomized controlled trial is highly unlikely to be undertaken. The study is also limited by selection bias as some women may have been poor candidates for operative vaginal delivery in general or for a certain type of operative vaginal delivery. The experience level of the provider is not known and regional differences among types of operative vaginal delivery offered cannot be accounted for. Similarly, 
Table 1 Baseline characteristics of women with prior cesarean delivery undergoing operative intervention in the second stage of labor

\begin{tabular}{|c|c|c|c|c|}
\hline & $\begin{array}{l}\text { Vacuum }(N, \%) \\
N=608\end{array}$ & $\begin{array}{l}\text { Forceps }(N, \%) \\
N=743\end{array}$ & $\begin{array}{l}\text { RCS (N, \%) } \\
N=486\end{array}$ & $p$-Value \\
\hline $\begin{array}{l}\text { Age } \\
\text { Mean (SD) }\end{array}$ & $29.5(0.24)$ & $29.0(0.21)$ & $29.9(0.25)$ & 0.247 \\
\hline \multicolumn{5}{|l|}{ Race } \\
\hline Non-Hispanic & $306(36.1)$ & $334(39.4)$ & $208(24.5)$ & \multirow[t]{4}{*}{0.003} \\
\hline Non-Hispanic black & $146(31.3)$ & $207(44.4)$ & $113(24.3)$ & \\
\hline Hispanic & $108(27.1)$ & $158(39.7)$ & $132(33.2)$ & \\
\hline Other & $48(38.4)$ & $44(35.2)$ & $33(26.4)$ & \\
\hline$\geq 1$ Prior vaginal delivery & $208(34.2)$ & $255(34.3)$ & $110(22.6)$ & $<0.0001$ \\
\hline $\begin{array}{l}\mathrm{BMI}>30 \\
\text { Mean (SD) } \\
N=235\end{array}$ & $34.11(0.43)$ & $34.97(0.53)$ & $34.99(0.54)$ & 0.258 \\
\hline Diabetes & $22(3.6)$ & $28(3.7)$ & $30(6.2)$ & 0.072 \\
\hline CHTN & $7(1.2)$ & $7(1.0)$ & $2(0.4)$ & 0.410 \\
\hline Pre-eclampsia & $20(3.3)$ & $22(3.0)$ & $23(4.7)$ & 0.435 \\
\hline $\begin{array}{l}\text { Gestational age } \\
\text { Mean (SD) }\end{array}$ & $39.6(0.046)$ & $39.6(0.042)$ & $39.8(0.052)$ & 0.140 \\
\hline
\end{tabular}

Abbreviations: BMI, body mass index; CHTN, chronic hypertension; RCS, repeat cesarean section; SD, standard deviation.

Note: Reference group is RCS.

Table 2 Maternal morbidity by planned mode of delivery in women undergoing trial of labor after prior cesarean delivery

\begin{tabular}{|l|l|l|l|l|}
\hline & $\begin{array}{l}\text { Vacuum }(N, \%) \\
N=608\end{array}$ & $\begin{array}{l}\text { Forceps }(N, \%) \\
N=743\end{array}$ & $\begin{array}{l}\text { RCS (N, \%) } \\
N=486\end{array}$ & $p$-Value \\
\hline Maternal composite morbidity & $148(24.3)$ & $240(32.3)$ & $107(22.0)$ & $<0.0001$ \\
\hline Uterine rupture & $5(0.8)$ & $8(1.1)$ & $19(3.9)$ & $<0.0001$ \\
\hline Surgical injury & $0(0)$ & $5(0.7)$ & $13(2.7)$ & $<0.0001$ \\
\hline Cystotomy & $0(0)$ & $4(0.54)$ & $12(2.5)$ & $<0.0001$ \\
\hline Enterotomy & $0(0)$ & $0(0)$ & $0(0)$ & NA \\
\hline Ureter injury & $0(0)$ & $1(0.13)$ & $1(0.2)$ & 0.569 \\
\hline Endometritis & $7(1.2)$ & $24(3.2)$ & $41(8.4)$ & $<0.0001$ \\
\hline Transfusion & $8(1.3)$ & $25(3.4)$ & $1(0)$ & 0.033 \\
\hline Peripartum hysterectomy & $2(0.3)$ & $0(0)$ & $2(0.4)$ & 0.318 \\
\hline Venous thromboembolism & $3(0.5)$ & $1(0.13)$ & $9(1.9)$ & 0.171 \\
\hline Wound complication & $2(0.3)$ & $3(0.4)$ & $0(0)$ & 0.006 \\
\hline Anal sphincter laceration & $94(15.5)$ & $169(22.7)$ & $0(0)$ & 0.012 \\
\hline Maternal death & $0(0)$ & $0(0)$ & NA \\
\hline
\end{tabular}

Abbreviations: NA, not available; RCS, repeat cesarean section.

some providers who offer both vacuum and forceps deliveries will offer a forceps delivery if the anticipated delivery will be more difficult due to maternal effort, station, or fetal position, and this is not accounted for in our analysis. This likely contributes to the increased maternal composite morbidity seen with forceps deliveries. Additionally, we excluded women with known mid-forceps deliveries but did not exclude women without a charted fetal station so likely some mid-station operative vaginal deliveries were included. While these deliveries are no longer recommended due to maternal and fetal adverse outcomes, they did occur during the time of data collection and could falsely elevate the incidence of adverse maternal outcomes in our cohort. Documentation of fetal station was only recorded at the time of admission, epidural placement, and maximal cervical dilation. Fetal station was not recorded at the time of cesarean delivery, and, without these data, we are unable to determine if these patients achieved a station where operative vaginal delivery would even have been a consideration. Finally, some maternal risks of RCD cannot be accounted for with this study but should be 
Table 3 Neonatal morbidity by planned mode of delivery in women undergoing trial of labor after prior cesarean delivery

\begin{tabular}{|l|l|l|l|l|}
\hline & $\begin{array}{l}\text { Vacuum }(N, \%) \\
N=608\end{array}$ & $\begin{array}{l}\text { Forceps }(N, \%) \\
N=743\end{array}$ & $\begin{array}{l}\text { RCS }(N, \%) \\
N=486\end{array}$ & $p$-Value \\
\hline Neonatal composite morbidity & $89(14.6)$ & $90(12.1)$ & $72(14.8)$ & 0.29 \\
\hline NICU admission & $68(11.2)$ & $60(8.1)$ & $58(11.9)$ & 0.322 \\
\hline HIE & $1(0.2)$ & $2(0.3)$ & $2(0.4)$ & 0.738 \\
\hline Apgar $<4$ at 5 min & $3(0.5)$ & $6(0.8)$ & $1(0.2)$ & 0.402 \\
\hline Perinatal death & $0(0)$ & $1(0.1)$ & $0(0)$ & 0.478 \\
\hline Birth injury & $17(2.8)$ & $21(2.8)$ & $11(2.3)$ & 0.812 \\
\hline
\end{tabular}

Abbreviations: HIE, hypoxic-ischemic encephalopathy; NICU, neonatal intensive care unit; RCS, repeat cesarean section.

considered such as maternal perceptions of failure and poor birth experience after laboring and needing a cesarean, increased risk of abnormal placentation in future pregnancies, increased degree of difficulty in future abdominal surgeries, and possible limitation on a woman's family size if she considers risks of future cesareans in this decision.

Operative vaginal deliveries were offered to significantly higher number of women with prior vaginal deliveries. This likely reflects the provider's preference to offer operative vaginal deliveries to those women who are at increased likelihood of having a successful vaginal delivery. Women who have had prior vaginal births have increased success rates of future vaginal deliveries. Choosing appropriate patients for an operative vaginal delivery is likely why the success rate of operative vaginal deliveries is high in this study. An opportunity for future studies could look at outcomes and success rates of women with and without prior vaginal deliveries.

Our analysis highlights the inherent risks of each type of operative delivery in the second stage. As is seen with all women undergoing operative intervention in the second stage, some risks are higher for operative vaginal delivery (e.g., perineal lacerations) and some are higher in the cesarean delivery group (e.g., surgical injury or wound complication); these risks are still present among women undergoing TOLAC with similar rates. As the neonatal outcomes are not significantly different, operative vaginal delivery and RCD are viable options in patients undergoing a TOLAC and the risks of each should be discussed with the patient to allow for shared decision making.

A great emphasis has been placed by governing bodies (American College of Obstetricians and GynecologistsSociety for Maternal-Fetal Medicine) to avoid the first cesarean section including redefinition of basic concepts such as active labor. This study supports that initiative one step later and places special attention in trying to prevent the second cesarean. Maternal morbidity only increases with repetitive cesarean sections, to this end, we believe that every effort made to avoid the second cesarean should be done, starting with offering TOLAC more readily and considering doing an operative vaginal delivery.

Conflict of Interest

None.

\section{References}

1 Hamilton BE, Martin JA, Osterman MJ. Births: preliminary data for 2015. Natl Vital Stat Rep 2016;65(03):1-15

2 Centers for Disease Control and Prevention (CDC). Rates of cesarean delivery-United States, 1991. MMWR Morb Mortal Wkly Rep 1993;42(15):285-289

3 Hamilton BE, Martin JA, Ventura SJ. Births: preliminary data for 2007. Natl Vital Stat Rep 2009;57(12):1-23

4 Silver RM, Landon MB, Rouse DJ, et al; National Institute of Child Health and Human Development Maternal-Fetal Medicine Units Network. Maternal morbidity associated with multiple repeat cesarean deliveries. Obstet Gynecol 2006;107(06): 1226-1232

5 Triebwasser JE, Colvin R, Macones GA, Cahill AG. Nonreassuring fetal status in the second stage of labor: fetal monitoring features and association with neonatal outcomes. Am J Perinatol 2016;33 (07):665-670

6 Martin JA, Hamilton BE, Osterman MJ, Curtin SC, Matthews TJ. Births: final data for 2013. Natl Vital Stat Rep 2015;64(01):1-65

7 Bailit JL, Grobman WA, Rice MM, et al; Eunice Kennedy Shriver National Institute of Child Health and Human Development Maternal-Fetal Medicine Units Network. Evaluation of delivery options for second-stage events. Am J Obstet Gynecol 2016;214 (05):638.e1-638.e10

8 Landon MB, Hauth JC, Leveno KJ, et al; National Institute of Child Health and Human Development Maternal-Fetal Medicine Units Network. Maternal and perinatal outcomes associated with a trial of labor after prior cesarean delivery. N Engl J Med 2004;351(25): 2581-2589

9 Son M, Roy A, Grobman WA. Attempted operative vaginal delivery vs repeat cesarean in the second stage among women undergoing a trial of labor after cesarean delivery. Am J Obstet Gynecol 2017; 216(04):407.e1-407.e5 